\title{
ImLib3D: An Efficient, Open Source, Medical Image Processing Framework in $\mathrm{C}++$
}

\author{
Marcel Bosc ${ }^{1,2}$, Torbjorn Vik ${ }^{1,2}$, Jean-Paul Armspach ${ }^{2}$, and Fabrice Heitz ${ }^{1}$ \\ 1 LSIIT UMR-7005 CNRS / Strasbourg I University, 67400 Illkirch, France \\ fabrice.heitz@ensps.u-strasbg.fr \\ 2 IPB UMR-7004 CNRS / Strasbourg I University, 67085 Strasbourg, France \\ \{bosc, vik, armspach\}@ipb.u-strasbg.fr
}

\begin{abstract}
ImLib3D is a $\mathrm{C}++$ library for 3D medical image processing research. It provides a carefully designed, object-oriented, standards conforming $\mathrm{C}++$ library, as well as a separate visualization system. Focus has been put on simplicity for the researcher who is considered to be the end-user. Source code is freely available and has been placed in an open collaborative development environment.
\end{abstract}

\section{Introduction}

The rapidly increasing complexity of medical image processing systems is a threat to the reproducibility of scientific work in this field. Reimplementing published work is often not feasible. Shared source code and shared development platforms are therefore becoming a necessity. Open source provides a well established framework for cooperative development, including high quality tools such as SourceForge.net. The ongoing success of systems such as Linux has proved that the Open Source model favors software quality. For the neuroscience community, Matlab ${ }^{\circledR}$ (mathworks.com) based Statistical Parametric Mapping (SPM: http://www.fil.ion.ucl.ac.uk/spm) is an important effort in this direction. However, ImLib3D, which is a stand-alone $\mathrm{C}++$ library, has inherent performance and software engineering advantages over Matlab based code. ImLib3D is an alternative to The Insight Toolkit (ITK: $i t k . o r g$ ), which also provides an interesting $\mathrm{C}++$ framework designed for 3D medical image processing.

ImLib3D (imlib3d.sourceforge.net) provides an object oriented, C++, 3D image processing library with a separate visualization system. The objective of the library is to create a a framework that motivates collaborative work, is simple to use and provides a coherent basis for research and development in medical image processing. The library is currently at a mature stage of development and is actively constructing a user base.

\section{Description of ImLib3D}

An overview of ImLib3D is shown in Fig. 1. The fundamental elements are the image classes that have been implemented as STL-like templated containers with 


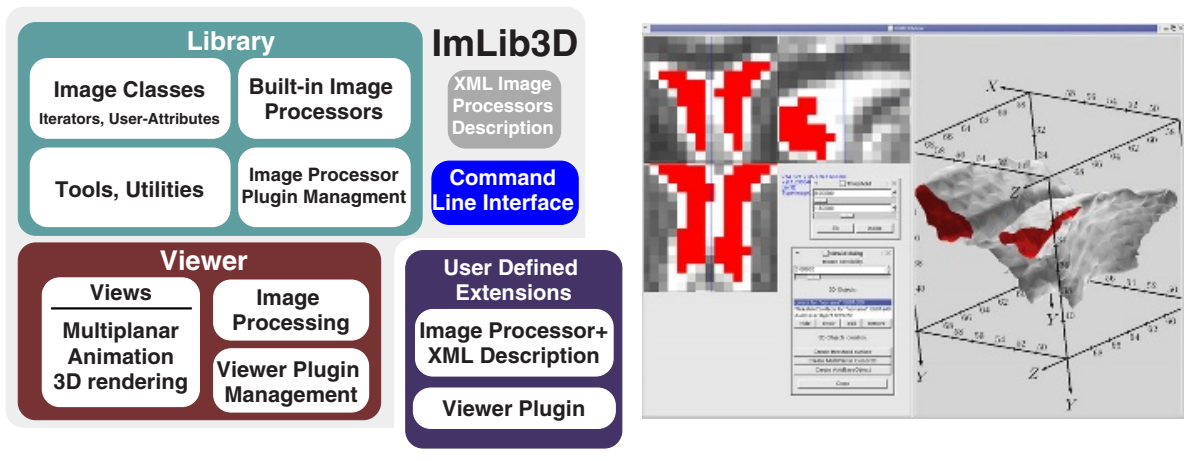

Fig. 1. (left) Overview of elements in ImLib3D. (right) Example of viewer rendering

STL-compliant iterators for traversing images or sub-images in various manners. It is therefore simple for the end-user to create images of arbitrary types, such as floating point images, complex-valued images, or deformation fields whose elements are $3 \mathrm{D}$ vectors. The use of templates also results in highly efficient code. Extensible attributes allow the user to dynamically add information to images, such as regions of interest, background values, and patient information.

ImLib3D defines a unified framework for describing image processing operators in XML format. Each operator has a full documentation as well as a precise description of argument types, allowing for automatic generation of fully documented viewer dialogs, command line interfaces, as well as dynamic extension with user defined image processors (image processor plugins). The commandline interface is particularly useful for high-level scripting, database interfacing and easy experimentation. Many low-level image processors are currently implemented: arithmetic, image-statistics (average, median, ...), morphological operations (erosion, distance transforms, connected component labeling, skeleton) with customizable structuring elements (neighborhoods), thresholding (simple, Otsu, ...), image transformations (affine or transformation field) with several methods of interpolation (including $\beta$-spline), linear filtering (arbitrary or separable convolutions, very fast Fourier domain filtering: fftw.org), image normalization and more.

The separate viewer features multi-planar ( 3 cross slices through a volume) views as well as surface rendering. Serial MRI may be visualized using animations. With the annotation system the user may interactively add and visualize textual or graphical information on images. The viewer is also dynamical extensible by user defined viewer plugins (dynamic libraries). 